INTERNATIONAL DESIGN CONFERENCE - DESIGN 2018

https://doi.org/10.21278/idc.2018.0195

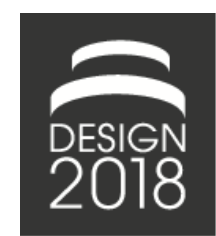

\title{
DISCURSIVE ENGINEERING DESIGN: A SPECULATIVE FRAMEWORK FOR DESIGNING TECHNOLOGICAL INNOVATION
}

\author{
C. B. Rebola and I. Gonsher
}

\begin{abstract}
The productive tensions between the sciences and the arts, between disciplines that make predictions and those that permit speculation, establish a creative dialectic. This paper describes a methodological approach for examining these kinds of creative dialectics and puts forth ways in which they might be implemented as educational models for cultivating innovation. The goal is to present a framework that builds on interdisciplinary pedagogy and hybrid experimentation - a technical and artistic rigor for approaching engineering and design as a mode of inquiry for innovation.
\end{abstract}

Keywords: product design, engineering design, design activities, case study, internet of things (IoT)

\section{Introduction}

The productive tensions between the sciences and the arts, between disciplines that make predictions and those that permit speculation, between engineering and design, establish and enhance a creative dialectic that generates new and innovative processes, practices, and products. Whereas science makes predictions, fiction, and the arts and humanities more generally, allow us to speculate, in order to imagine scenarios that do not yet exist. This paper describes a methodological approach for examining these kinds of creative dialectics, and puts forth ways in which they might be implemented as educational models for cultivating innovation within the classroom, studio, and lab. The goal of this paper is to present a framework that builds on interdisciplinary pedagogy and hybrid experimentation - a technical and artistic rigor that approaches engineering and design as a mode of inquiry that produces innovative outcomes.

This paper presents a case study of two classes, from two adjacent but very different institutions, one grounded in the arts and the other grounded in the sciences. These classes shared the common goal of challenging students to envision a future where emerging technologies will reshape everyday experiences in ways that will enhance our humanity rather than robbing us of it.

Designers and engineers in the 21 st century will need to better understand the context they are designing for as they apply empiric insights toward human needs. This will demand an evolution in design and engineering pedagogy. We need to better prepare students to ask both "how" and "why", as they embrace a multidisciplinary approach to creative problem finding and problem-solving. This interdisciplinary approach is necessitated by an increasingly complex world that demands increasingly complex solutions to increasingly complex problems.

\section{Science in design}

In nearly every iteration of our engineering design classes, in a room filled with engineering and design students ready for a semester of collaboration, the same question always emerges: Who is primary: the engineer or the designer? 
Engineers, by training and temperament, typically tend to first identify and fix constraints, as they work to optimize solutions within those constraints. It is a process that privileges certainty over ambiguity, convergent thinking over divergent thinking, and optimization over heuristics. Convergent thinking is the ability to frame a problem in such a way as to produce a single, correct answer. By contrast, designers tend to begin with divergent thinking, which is a process for generating as many solutions as possible, reframing and transcending constraints (Guilford, 1967). Divergent thinking is open-ended, often ambiguous, and easily recognizable in common design strategies for ideation and brainstorming. The mantras of Design Thinking popularized by IDEO - "defer judgment" and "build on the ideas of others" - are manifestations of divergent thinking (IDEO U, 2016).

The anthropologist Claude Lévi-Strauss offered another similar way to understand the relative advantages and disadvantages of these strategies within the creative process. He compared the engineer with the bricoleur. A bricoleur is a person who makes due with what is at hand, open to discovering unexpected and novel ways of using the available material to solve a given problem. An engineer, by distinction, works to create what she or he needs from scratch, in an efficient and optimized manner. This typically demands a well-defined problem, with clearly delimited constraints, within which the creativity of the engineer operates. Lévi-Strauss describes the distinction in the following way:

"The 'bricoleur' is adept at performing a large number of diverse tasks; but, unlike the engineer, he [or she] does not subordinate each of them to the availability of raw materials and tools conceived and procured for the purpose of the project. His [or her] universe of instruments is closed and the rules of his [or her] game are always to make do with "whatever is at hand," that is to say with a set of tools and materials which is always finite and is also heterogeneous because what it contains bears no relation to the current project, or indeed to any particular project, but is the contingent result of all the occasions there have been to renew or enrich the stock or to maintain it with the remains of previous constructions or destructions." (Lévi-Strauss, 1962)

\subsection{Bridging the gap}

The answer we usually eventually arrive at in class to the question posed by the students about the nature of the process, and which discipline to privilege over the other, is that you need multiple approaches, but every project may require a different balance. You need to be both a designer and an engineer. You need to think divergently at some points in the process, while at other times convergent thinking is necessary. The trick is in knowing, intuitively and with discipline, when which strategy is appropriate given its place in the process and the context one is designing for. This is why we emphasize critical discussion about process and documentation in our classes, so that students might be better attuned to their creative practices, and the outcomes they produce.

Another way to think about this creative dialectic between divergent thinking and convergent thinking, between being open or closed to possibilities, between saying 'yes' or 'no', is to consider one's own critical perspective as an intimate part of the process. When we are in the flow of our work, fully immersed in what we are creating, students will often discover creative insights that move the process forward. However, sometimes we can be too close to our work, too myopic and blinded to other possible solutions, and therefore stepping back for a bit of critical distance, and bringing in the perspectives of others, gives us a vantage point from which to cultivate deeper insights. The documentation of this process is one of the most important pedagogical tools we have to accomplish this. Documentation accomplishes several important things: 1) It provides a record to help students establish a portfolio of work that demonstrates project-based learning. 2) It allows students to share their work with peers and collaborators. 3) But perhaps most importantly, it gives students critical tools to more deeply understand their process.

Students in our classes are often given tremendous creative freedom to reframe projects in ways that align with their understanding of the context. But this freedom also comes with the responsibility to consider why they make certain design decisions throughout the process. Documentation is what gives them the tools to do this. Documentation allows them to clearly see the evolution of the project, and to better understand the arc of the process. Because the design process is iterative, there is room to explore and make mistakes, while still making forward progress towards a final outcome.

Often the engineers in the class will adopt the first idea they have, and work towards implementing that idea in the most expedient way. We have found that by documenting the iterative design process, it gives 
students more design possibilities to draw on, more ways to frame and address a problem, and takes the burden away of investing too much in one solution too early in the process. How can you know if it is the best solution if you haven't considered others to compare it to?

This iterative approach to the process can be thought of as a series of experiments. Design strategies such as Design Thinking can be thought of as the scientific method applied to the creative process. Hypothesize, experiment, and analyze become understand, prototype, and critique, in the parlance of designers.

But the process cannot remain open-ended, which is why we need convergent thinking too. As Dym et al. (2005) point out, "the design of systems is rarely accomplished exclusively by applying fundamental scientific principles. In most cases, the design of systems also requires some use of empirical data and experimentation. This fact is driving a trend to teach engineers the design of experiments so they can more efficiently plan experiments and analyze and understand the results."

Every iteration is an opportunity to ask a new question. Each project is an experiment to deepen knowledge, which might be applied towards some human-centered goal. Design and engineering are modes of inquiry, which is enhanced by the cultivation of curiosity in students. By giving students better tools to navigate these creative dialectics, through critical engagement with process, practice, and product, we plant the seeds for the innovative leaps of tomorrow.

\section{Discursive engineering design education}

This paper presents a case study of a collaboration between two classes from two different institutions grounded in the arts and engineering, the Rhode Island School of Design and Brown University School of Engineering, that joined efforts and build on their distinctive strengths. We will refer to these classes as the "Engineering Skills Class" (E) and the "Design Skills Class" (D). The classes shared the common goal to envision the future of telepresence robotics under the discursive and speculative design approach. The topic was chosen based on the expertise of the instructors while providing ample opportunities for students for the approach. And the approach was formulated by the instructors to exercise skills for design and prototyping technologies while primarily focusing on speculative-future creative applications.

The starting of the class was based on the assumption that there is a need to question and explore better opportunities for an everyday life that is saturated with a dazzling array of interconnected, automatic things. The classes were tasked to question the "how" and "why" of designing robots (and robot-like things) and focused on exploring the role of emerging technologies impacting our lives in the near future with the design, discussion, and fabrication of robots.

One of the challenges of the collaboration was to maintain the curricular goals for each of the classes. The Engineering class goal was to introduce students to digital technologies (Arduino, Raspberry Pi's) as well as the creative uses of off-shelf technologies (i.e. drones, projectors). The goals for the class was not only to "fabricate" technologies but mainly doing it meaningfully. Much of the class was focused on a methodology to allow questioning applications within a human-centered design lens. The constraints for the class was to design and build a robotic technology speculative application and address the following: 1) the telepresence robot must afford communication at a distance using a technology; and 2) the telepresence robot must move in space.

The Design class goal was to introduce students to discursive design as well as bridging the gap between ideation and fabrication while maintaining a critical perspective in the creative process (Hermano and Somerson, 2013). Students were tasked to move from ideas to concrete propositions, a robot as an outcome, but questioning the current conventions of what exists today and proposing a discursive proposition via a working prototype.

Discursive design is one of the four fields for practicing Industrial Design (Tharp and Tharp, 2009). This field is rooted in practicing design from a critical perspective by proposing tangible outcomes that promote critique and conversation (Dunne and Raby, 2013). Tharp and Tharp (2015) further explain that:

"The primary goal is to prompt self-reflection, ignite the imagination, and foment contemplation - to deliberately make the user think (deeply)...the product is given form and function so that it can communicate ideas - this is the goal and the measure of success. Rather than tools for living and doing, these are tools for thinking". 
Within the discursive design lens, both classes used the following keywords as variables for the projects: embodiment, presence, and communication (modes/directions). In any of these concepts students would question through exploration:

- What is it?

- How is represented today? Can I gather quick evidence in my current environment?

- Can I build a visual taxonomy of behaviors associated with it?

- What are science fiction sources (including magazines) that can illustrate it?

- How would these issues be manifested intrusion/facilitation/control/augmentation?

- How about these human properties: humor/energy/rituals/curiosity/optimism?

- What if a day without it?

- What are the negative connotations with the concept?

Central to this project was the task of experimentation. Materials representations were a necessity for the project for both development and decision-making. However, the practice of storytelling, both in a narrative and visual manner was also essential for the process. Storytelling allows explorations to become tangible and understandable in the process of designing (Saffer, 2010; Greenberg et al., 2012). It turns ideas into concrete and evaluative instances for future design decision-making in the process. Students were asked to generate storytelling and performative pieces (scenarios, time-based pieces, essays) as experimentations to construct the discursive reality. They were given speculative storytelling examples such as the film artworks of Julian Prévieux $(2014,2016)$.

\subsection{Collaborative framework}

One of the first steps in the collaboration was to generate a collaborative framework to guide the class expectations and interactions. The framework emphasizes the need of process built from the arts and technical rigor in addition to outcomes for bringing about innovative propositions. Innovation in our case study is the fact that we merged two classes, two different realms that were dealing with same terminologies but different definitions (see Table 1). While the joint class will have the same end goal: envisioning the future of telepresence robotics, our framework shows that we brought together two different views on the topic within the same pedagogy. Even though the classes would work within an integrative approach, the framework would emphasize one side with skills for prototyping technologies, and another focusing on the speculative creative application, as follows:

Table 1. Discursive engineering design framework

\begin{tabular}{|l|l|l|}
\hline & E Focus & D Focus \\
\hline Phase 1 & $\begin{array}{l}\text { Movement } \\
\text { (Identify Tech) } \\
\text { (Tech Centric) }\end{array}$ & $\begin{array}{l}\text { Human Purpose } \\
\text { (Identify Issue) } \\
\text { (Human Centric) }\end{array}$ \\
\hline Phase 2 & Experiment Sensing & Experimental Environmental opportunities \\
\hline Phase 3 & Experiment Structure & Experiment Tangible Manifestations \\
\hline Phase 4 & Experiment Control & Experiment Connectedness \\
\hline
\end{tabular}

\subsubsection{Phase 1}

This phase required students to explore opportunities from the technical and human perspective. Students were tasked to identify technologies to play with (i.e. Arduino) as well as identify issues of telepresence (see Figure 1 and Figure 2). Using the questions listed in section three, students engaged in brainstorming potential issues. Some of the issues included: envisioning the future of relationships mediated by robots; a world where robots are bad; relationship robots for companionship and comfort; communicating with the dead; communicating with different languages such as plants; disembodiment of the self in other beings such as dogs; to mention a few. 


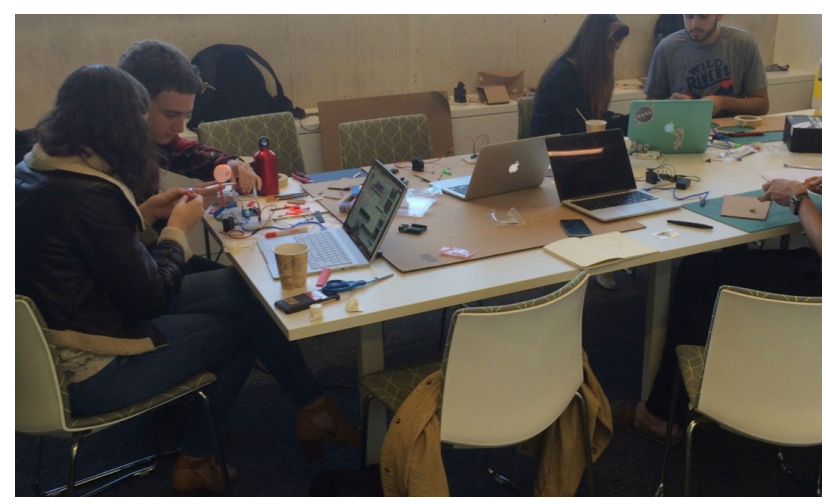

Figure 1. Technical explorations

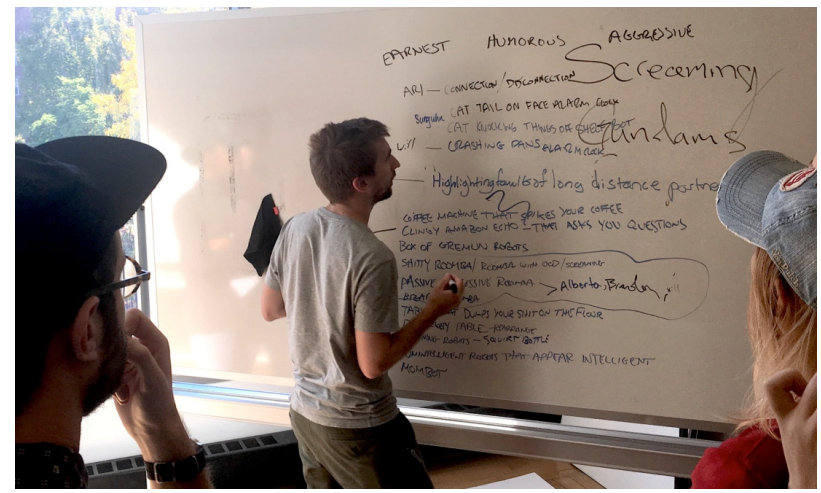

Figure 2. Issues identification

\subsubsection{Phase 2}

As a second phase, students were tasked to move from the issue and technical opportunities to a larger scale. In the case of technical explorations, students started to consider the role of sensing technologies for enhancing the selected technology. Projects would make use of proximity sensors to allow the robots to navigate space autonomously. They would also consider adding other sensors as well and explore how robots can be sensitive to the environment. Simultaneously, students were also tasked to bring the human-centered issue in context by identifying environmental opportunities. Environmental opportunities refer to the role of an entity in the environment in order to deconstruct the product to a situation and location. During this phase, students develop their design thinking by discussing and sketching ideas. They made mock-ups of first iterations, that "tell the story" of how these robots might work. Mockups were developed with readily available technology and materials and prototyped propositions with scale models and enactments (see Figure 3 and Figure 4).

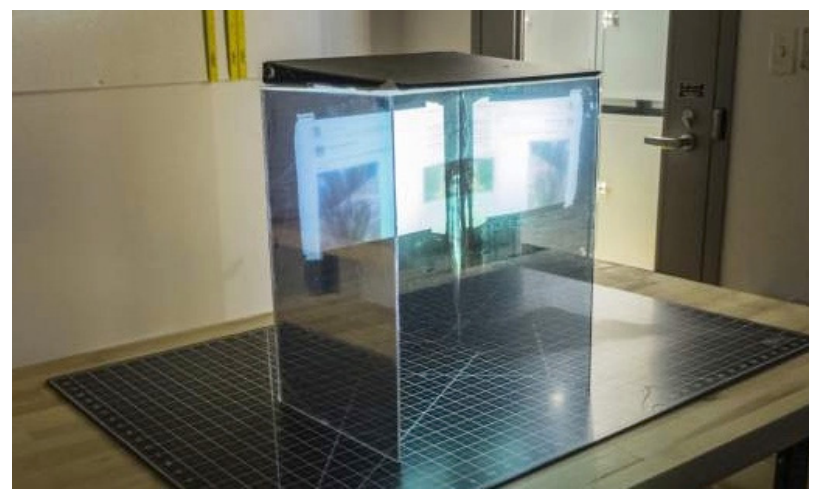

Figure 3. Environmental explorations 


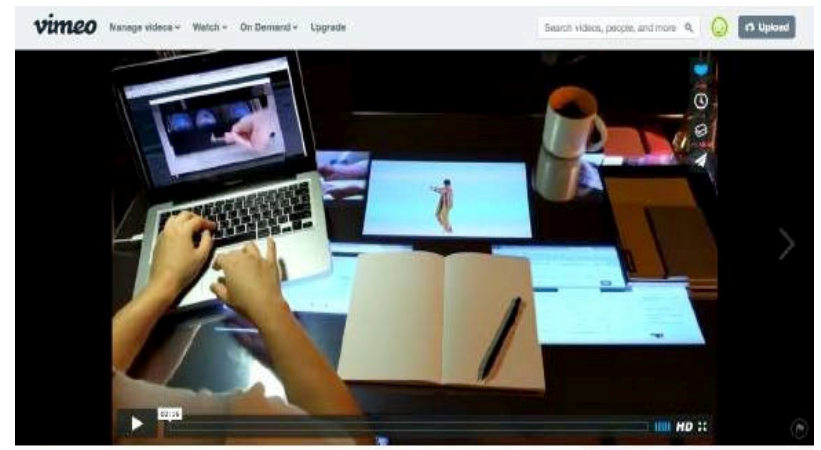

Observation Workspace

Search rasults for "osservation
warkenacen"

Figure 4. Issues development

\subsubsection{Phase 3}

During this phase, students elevated their propositions by considering structures as in functional and tangible manifestations. This phase required students to integrate explorations by considering ways the future robots would integrate the environment with a tangible form. It was also a phase to experiment with how the propositions would meet user needs.

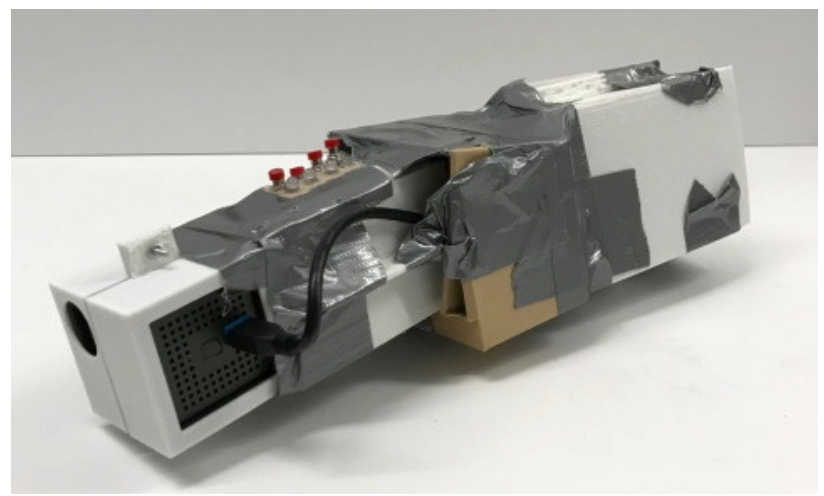

Figure 5. Experimenting with prototypes

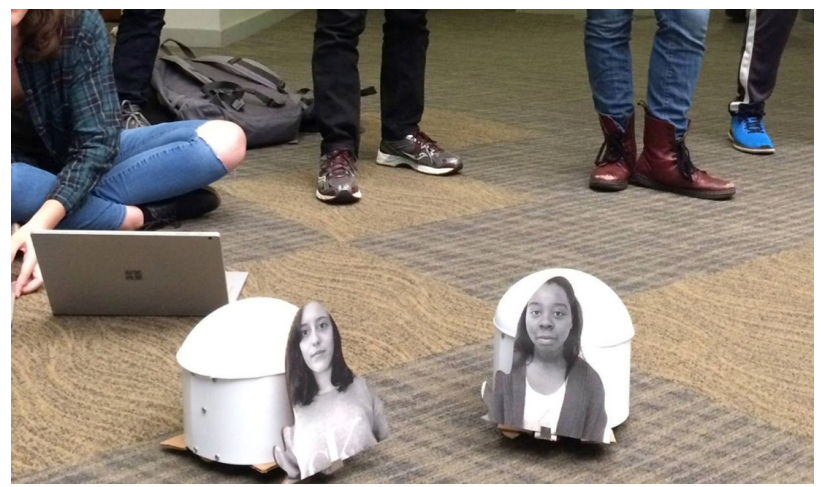

Figure 6. Experimenting with behaviors

\subsubsection{Phase 4}

In this last phase of our framework, projects needed to exercise environmental control and connectedness. Within the iterations developed in earlier phases, environmental control was referred as to considerations of the robot's technical aspects regarding affordances for the given-designed behavior. Similarly, projects also needed to evolve in terms of connectedness, integrating behaviors of 
the technologies, within environments (local and distant) as well as user behaviors. The experimentation on connectedness would respond to the "ecology" requirements of the projects, meaning how the design interventions live in our built and human environment. All phases together would respond to the general rationale of the class perspective, based on the following resulting formula:
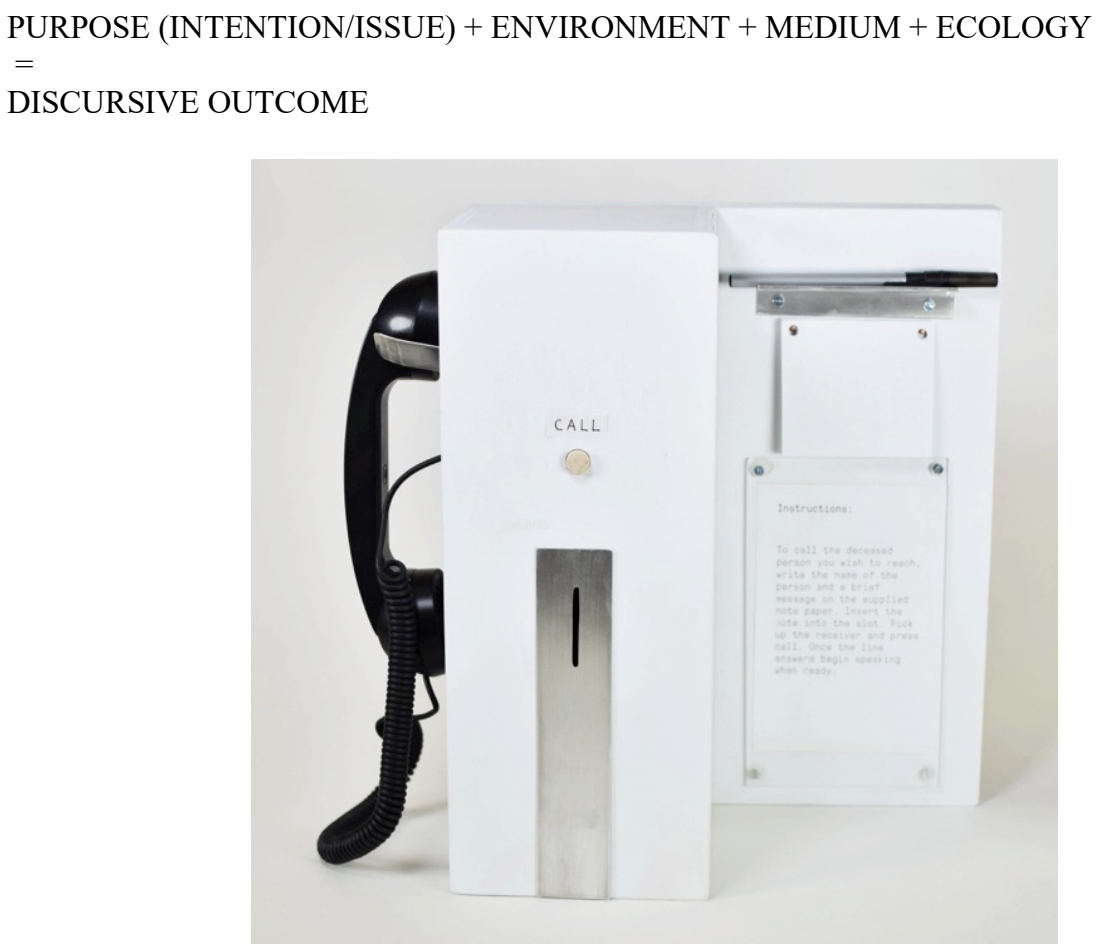

Figure 7. Mourning phone proposition

\subsubsection{Result examples}

As a final phase, projects were further refined for implementation. During Phase 4, projects received expert and class critiques that were incorporated in the final refinement. Figure 8 showcases a design proposition "Portal Gun". Inspired by the popular video game, this live-action version takes players on the wild scavenger hunt throughout the public space on campus, unlocking clues that provide portals to other places where players find more clues that unlock the puzzle. Figure 9 showcases a project that was inspired by attempts earlier in the phases to get chatbots to talk to and empathize with each other. Using the current Facebook icons and movement (running away or running towards someone) the robot's emotional valence was expressed. This project provokes questions about the emotional lives of robots, and how we as humans empathize with intelligent objects.

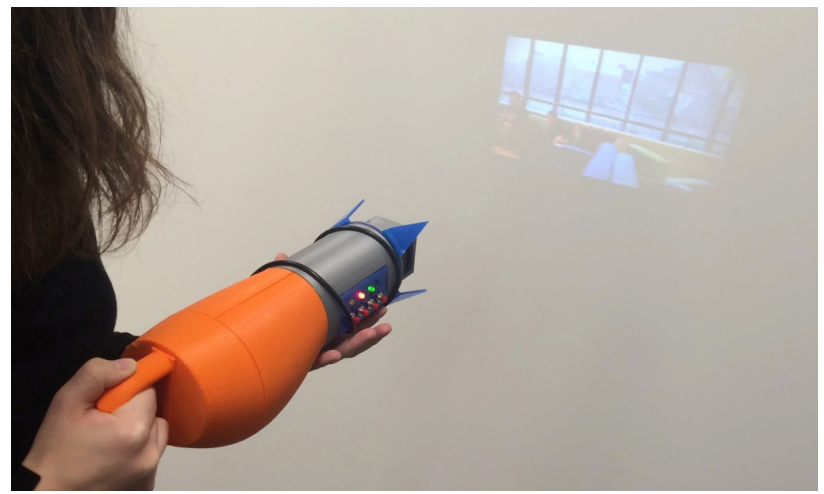

Figure 8. Portal gun 


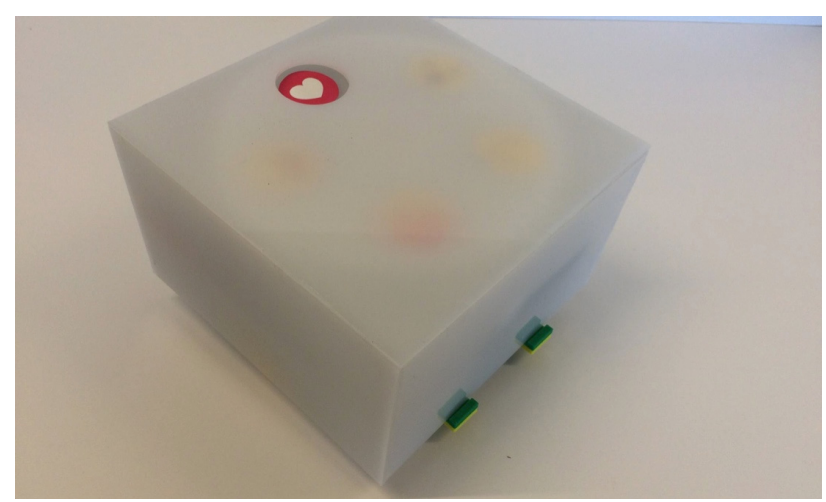

Figure 9. Emotional bot

\section{Discussion}

Overall, the results of the collaboration were successful and diverse. Outcomes were insightful both technically and critically, exercising our discursive engineering design framework. While the collaboration was positive, there is room for improvement summarized in three main points: open/close relationships, finding why/how balances, and critique of the critique. The next sections explain in detail each of these points.

\subsection{Open/close relationships}

Outcomes of the collaboration reflected the use of varied processes and practices across teams. The most successful teams were the ones rejecting linear processes where the design criteria were defined too early and too general, closing off possibilities for further experimentation and exploration, placing too much of an emphasis on convergent thinking at the expense of divergent thinking. We proposed a framework that encouraged a balance between abstract procedures and concrete explorations; between divergent and convergent thinking. Keeping projects open-ended (procedures) yet focused and concrete (context) can help students find the proverbial needle (awesomeness) in the haystack.

These modalities were also apparent with regard to the manner in which the teams collaborated on their projects. The question arose of how best to leverage the diverse insights of the team (divergent thinking) while synthesizing and bringing together those insights into a cohesive whole (convergent thinking). The multidisciplinary, collaborative nature of the teams ensured that each team found a different balance of these elements given the context of their project.

Multidisciplinary collaborative projects often face the challenge of bringing the design elements together and leveraging an optimal blend of expertise. Innovative ideas can be discarded too easily by the preconceptions of "experts", and individuals can become protective of their processes affecting the team performance. A willingness to be open to new possibilities is therefore necessary to cultivate within the culture of the team. However, there was also the need to temper this by encouraging teams to converge on an appropriate solution to the problems they defined through their process of experimentation and prototyping.

\subsection{Finding why/how balances}

Our experience with the collaboration highlighted the importance of balancing the "why" and "how" on thematic constructs to foster innovative design. While the "how" questions helped define the technical constraints of the project, the "why" questions helped define the purpose and context. In our case, due to the nature of the institutions, i.e. RISD's somewhat agonistic "art school" culture, and Brown Engineering's culture of designing for optimization, the creative process was challenged by "artistic explorations"-self-indulgence and technically driven approaches. While both approaches are necessary, a balance should be present, especially when considering a bias towards thinking and doing. Meaning, there should be a balance of problem-setting and problem-solving activities. One way to resolve the balance could be incorporating a synthetic approach. This synthetic approach could be based 
on systematic experimentation both "artistic" and technical experimentation focusing on process rather than outcomes. Yet, having integrative checkpoint along the process (i.e. critique). While our proposed framework can allow such experimentation, it failed to specify routes for experimentation and methods for integrating explorations. Future framework applications will need to further refine and develop these systems to exercise a better balance between "why" and "how".

\subsection{Critique of the critique}

Design disciplines are built around a culture of critique. Critiques are a crucial component for design and development of propositions by giving students the opportunity to step back and consider the project in a broader context. While the effect of critique is usually positive in most cases, offering new paths for innovation and the development of a project, the critique itself can be seen as "negative" by the students. This emphasis on the negative aspects of the design is often deployed to help students recognize unsuccessful design routes. And yet, too often, we find a bias towards "no" (negation) produce a negative climate within the design studio, sometimes leading to a general distrust of others' motives. This can create a cynical and somewhat toxic environment that can be paralyzing to the creative process. Future refinements of our framework should suggest modes of critique that give students both an understanding of the limitations of their design, while framing the feedback positively, building upon rather than tearing down, and always offering suggestions for further improvement (solution focused). These solutions may be prescriptive, in terms of offering suggestions for improvements to particular features of the design, or process-oriented, offering new questions to consider or further experimentation and research to explore.

\section{Conclusion}

Collaborations across engineering and design still remain a challenge for innovation. The boundaries between these modes of creative problem solving can be fluid and mutable in practice, and often are, but there are still significant differences in how engineers and designers are trained, and how this training provides different vantage points for creative problem solving. This paper presented a case study of two classes, from two different institutions grounded in the arts and engineering, that joined efforts and build on their distinctive strengths. We proposed a discursive engineering design framework for collaboration built on the strengths of the institutions. The framework emphasized the need for process built from the arts and technical rigor in addition to outcomes for bringing about innovative propositions within the same pedagogy. We have documented our experience with the hope that it will inspire others in the engineering and design community to practice the collaborative experiences fostering innovative technological development and applications. Overall, the results of the collaboration were successful and diverse. Outcomes were insightful both technically and critically, exercising our discursive engineering design framework. While the collaboration was positive, there is room for improvement focusing on open/close relationships, finding why/how balances, and critique of the critique. Yet, the presented framework proved to facilitate and create conditions for advancing conversations and practices across polar disciplines. We need to stop being cynical and critical to disciplines but issues centered around impacting innovation with propositions that are engineered with the design. There is a need to exercise both linearity and chaos in the process if we want to deliver propositions that respond to critical issues.

\section{Acknowledgments}

We would like to thank the Humanity Centered Robotics Initiative (HCRI) at Brown University, and the Industrial Design Department at the Rhode Island School of Design for supporting collaborations between institutions. Special thanks to Peter Haas, Associate Director of HCRI for helping fund the technologies for the collaborative projects.

\section{References}

Dunne, A. and Raby, F. (2013), Speculative Everything: Design, Fiction, and Social Dreaming, The MIT Press, Cambridge, Massachusetts. 
Dym, C.L., Agogino, A.M., Eris, O., Frey, D.D. and Leifer, L.J. (2005), “Engineering Design Thinking, Teaching, and Learning", Journal of Engineering Education, Vol. 94 No. 1, pp. 103-120. https://doi.org/10.1002/j.21689830.2005.tb00832.x

Greenberg, S., Carpendale, S., Marquardt, N. and Buxton, W. (2012), Sketching User Experiences: The Workbook, Morgan Kaufmann Press, Waltham, Massachusetts.

Guilford, J.P. (1967), The Nature of Human Intelligence, McGraw-Hill, New York.

Hermano, M.L. and Somerson, R. (2013), The Art of Critical Making: Rhode Island School of Design on Creative Practice, John Wiley \& Sons, Hoboken, New Jersey.

IDEO U (2016), Effective Brainstorming Techniques. [online] IDEO. Available at: http://www.ideou.com/pages/brainstorming

Lévi-Strauss, C. (1962), The Savage Mind, University of Chicago Press, Chicago.

Prévieux, J. (2014), What Shall We do Next? (Sequence \#2). [online] Jousse Entreprise. Available at: https://vimeo.com/111013619

Prévieux, J. (2016), Julien Prévieux. Expositions, Publications, Portfolio. [online] Julien Prévieux. Available at: http://www.previeux.net

Saffer, D. (2010), Designing for Interaction: Creating Innovative Applications and Devices, 2nd ed., New Riders, Pearson Education, Berkeley, California.

Tharp, B. and Tharp, S. (2009), The 4 Fields of Industrial Design: (No, Not Furniture, Trans, Consumer Electronics, \& Toys). [online] Core77 Inc. Available at: http://www.core77.com/posts/12232/the-4-fields-ofindustrial-design-no-not-furniture-trans-consumer-electronics-toys-by-bruce-m-tharp-and-stephanie-m-tharp12232

Tharp, B. and Tharp, S. (2015), What is Discursive Design? [online] Core77 Inc. Available at: http://www.core77.com/posts/41991/What-is-Discursive-Design

Dr. Claudia B. Rebola, Associate Professor and Graduate Studies Coordinator

School of Design, University of Cincinnati, Industrial Design

342 Clifton Ct, 45221 Cincinnati, United States

Email: claudia.rebola@uc.edu 